\title{
PROBABILISTIC LIFE OF DA718 FOR AIRCRAFT ENGINE DISKS
}

\author{
S.DEYBER ${ }^{1}$, F.ALEXANDRE ${ }^{2}$, J.VAISSAUD $^{2}$, A.PINEAU $^{2}$ \\ ${ }^{1}$ Snecma Materials \& Process Engineering \& Quality Division, \\ 77550 Moissy-Cramayel, France \\ ${ }^{2}$ Ecole des Mines de Paris - Centre des Matériaux P-M Fourt, \\ BP87, 91003 Evry Cedex, France
}

Keywords: Direct-aged IN718, crack initiation, probabilistic model

\begin{abstract}
The micro-mechanisms of crack initiation and propagation identified for DA 718, as well as the competition between initiation site types and locations, cannot be accurately accounted for by current certified methods used to establish aircraft engine disks life,essentially phenomenological.

Once identified, these micro-mechanisms have been modelized by mesoscopic models using very few parameters and depending strongly on material mechanical behavior. LCF data scatter was mainly attributed to probabilistic nature of particle presence in a given volume, and probability of fracture at the first LCF cycle.

In order to describe these effects and develop a model able to account for LCF scatter by the only means of physical mechanisms description, a global macroscopic and probabilistic model has been proposed. This model can be used as a post-processing routine of a finite element analysis.

Confrontation of the global probabilistic model to experimental data gathered on notched samples gives good results, and the proposed method shows more realistic than the standard one on the cases exposed. Contrary to the standard method, it allows in addition to determine the predominant initiation site as a function of loading, material behavior and microstructure inherited from forging process, giving way to possible material and forging route optimisation at the design stage, to fulfill given structural life objectives.
\end{abstract}

\section{Introduction}

The current certified methods used to establish aircraft engine disks life are essentially phenomenological, and based on numerous fatigue tests. A statistical approach based on standard deviation is applied to fatigue test results to take into account dispersion and determine minimum life to initiation. Such methods are empirical and conservative, but cannot adapt easily to a change in materials or processes, and can be over-conservative, especially in stress concentration areas.

As a guide for materials and design engineers, an alternative approach is proposed. It is based on microscopic crack initiation and propagation mechanisms identified in IN718 through appropriate testing: micro-crack initiation on second phase particles (carbides or nitrides) and grains, and micro-crack propagation. These mechanisms, observed for direct-aged IN718 (DA 718) used by Snecma for turbine disks, are summarized in the first section below. 
The second section deals with life models developed and identified to take into account microstructure and mechanical behaviour inherited from IN718 disk forging process. The combination of these microscopic models allows, at a macroscopic level, the description of the competition of various crack initiation sites observed in IN718 alloy. The macroscopic method described here is a probabilistic one, which allows design engineers to eventually determine disk life for a given probability of fracture.

In the third and last section, the proposed probabilistic life method is validated by confrontation to notched fatigue experimental results obtained on DA 718. Experimental data gathered, finite element simulations conducted and life results are presented.

\section{Crack initiation and propagation mechanisms in DA 718}

The aim of this section is to summarize the main characteristics of crack initiation and propagation observed in direct-aged IN718, used in forged turbine disks. This alloy is widely used and has been thoroughly studied: we focus here on observations carried out at Ecole des Mines de Paris [1-3] on Snecma material.

\section{$\underline{\text { Material }}$}

The nominal composition of Inconel 718 alloy is: $19 \% \mathrm{Cr}, 18 \% \mathrm{Fe}, 5 \% \mathrm{Nb}, 3 \% \mathrm{Mo}, 1 \% \mathrm{Ti}, 0.5$ $\% \mathrm{Al}, 0.05 \% \mathrm{Ta}, 0.05 \% \mathrm{C}$, balance $\mathrm{Ni}$ (weight \%). This material is an austenitic FCC $\gamma$ matrix strengthened by the precipitation of $\gamma^{\prime}\left(\mathrm{L}_{2}, \mathrm{Ni}_{3}(\mathrm{Ti}-\mathrm{Al})\right)$, and $\gamma^{\prime \prime}\left(\mathrm{DO}_{22}, \mathrm{Ni}_{3}(\mathrm{Nb}-\mathrm{Ti})\right)$ particles. The volume fraction of $\gamma^{\prime}+\gamma^{\prime}$ ' particles is about $20 \%$ [4].A third type of precipitate is also present in the alloy which is the $\mathrm{Ni}_{3} \mathrm{Nb} \delta$ (DOa) phase. The $\delta$ phase particles are located both along the grain boundaries and within the grains, depending on the heat treatment applied to the alloy. Another family of second phase particles present in the alloy are nitrides (TiN) and carbides $(\mathrm{NbC})$ with a size ranging from 5 to $20 \mu \mathrm{m}$. These $\mathrm{TiN}$ and $\mathrm{NbC}$ particles are preferentially located at grain boundaries.

This wrought material is usually produced by forging followed by different kinds of heat treatment in order to obtain the desired microstructure, in particular small grain size microstructure which is assumed to be beneficial for mechanical strength and low cycle fatigue (LCF) properties. The most widely used heat treatment is that corresponding to annealed and aged conditions. Annealing is usually performed at $955^{\circ} \mathrm{C}$ below the solutionizing temperature of the $\delta$ phase. This annealing treatment enables to control the grain size. Conventional ageing is performed at $720^{\circ} \mathrm{C}-8$ hours followed by cooling at $50^{\circ} \mathrm{C} / \mathrm{hr}$ and final ageing at $620^{\circ} \mathrm{C}$ for 8 hours. More recently significant improvements have been made to reduce the grain size of this material. This was mainly achieved by using a thermomechanical heat treatment, referred to as Direct Ageing (DA); see e.g. [5-7]. In this treatment the material is directly quenched after forging and subsequently aged at $720^{\circ} \mathrm{C}+620^{\circ} \mathrm{C}$. Resulting grain size ranges between $5 \mu \mathrm{m}$ and $10 \mu \mathrm{m}$, and more substantial hardening is achieved than in the case of the conventional annealing and ageing heat treatment. The material used for experimental data presented here is DA 718.

\section{Fatigue Crack Initiation Sites}

The LCF properties of this material have been extensively investigated (see e.g. [1, 3, 8-10]). The effect of grain size reduction ,assumed to be beneficial on fatigue lives, was more particularly studied for conventional and DA 718 alloys (see e.g. [1, 2, 10]). These studies confirmed that the reduction in grain size produces a significant increase in fatigue life, as shown in Figure 1, where the material is tested at $350^{\circ} \mathrm{C}$ and a frequency of $1 \mathrm{~Hz}$ under strain controlled 
conditions $\left(R \varepsilon_{\mathrm{t}}=\varepsilon_{\min } / \varepsilon_{\max }=0\right)[10,3]$. These fatigue studies also revealed that for very low grain size (lower than $10 \mu \mathrm{m}$ ) obtained by DA heat treatment the results of fatigue life measurements were largely scattered. In this range of grain size the shortest fatigue lives were systematically associated with a modification in the nature of fatigue crack initiation sites. At large grain sizes typical transgranular stage I crack initiation along intense slip bands was observed $[3,11]$. On the other hand, when the grain size was smaller than about $10 \mu \mathrm{m}$, fatigue cracks were preferentially initiated at second phase particles, such as niobium carbides ( $\mathrm{NbC}$ ) or titanium nitrides (TiN) [3]. This produced a significant decrease in LCF life, as observed in Figure 1.

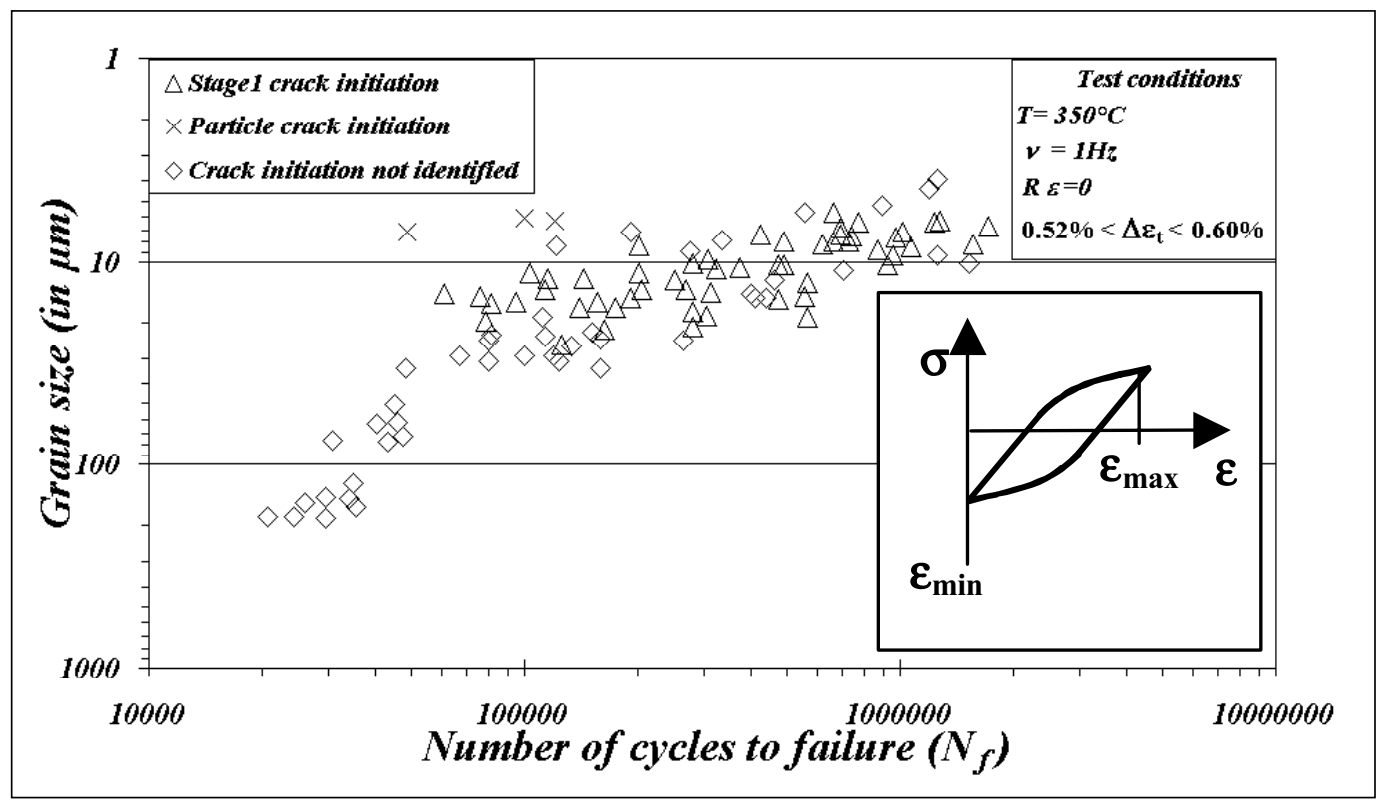

Figure 1. Fatigue grain size vs. number of cycles to failure in relation with crack initiation types $[3,10]$.

Figure 2 illustrates these two types of initiation sites, intense slip bands (stage I ) and second phase particles, for various heat treatments and resulting grain sizes [3].

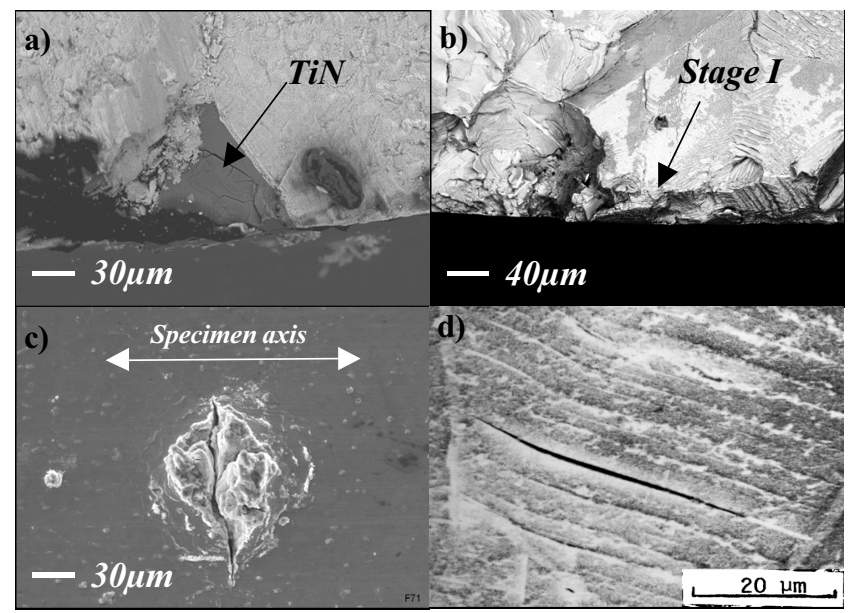

Figure 2. Fatigue crack initiation sites observed in IN718:

a) titanium nitride in a 5-10 $\mu \mathrm{m}$ grain size material, b)intense slip bands (Stage I) in a 150 $\mu \mathrm{m}$ grain size alloy, c) niobium oxide on the gauge length of a 5-10 $\mu \mathrm{m}$ grain size material, d) Stage I on the gauge length of a $150 \mu \mathrm{m}$ grain size alloy $[1,3]$.

Detailed studies [1] showed that in the case of crack initiation on particles, initiation can occur on sub-surface particles instead of surfaces particles; the competition between surface and sub- 
surface sites can be attributed not only to effects of crack propagation rates and thresholds in short cracks (see e.g. [12]), but also to the probabilistic nature of second phase particle presence and crack initiation on particles. These aspects have been precisely studied through in situ tensile tests carried out on DA718 specimens placed in a SEM observation chamber [2]. The results of such experiments are illustrated in Figure 3.

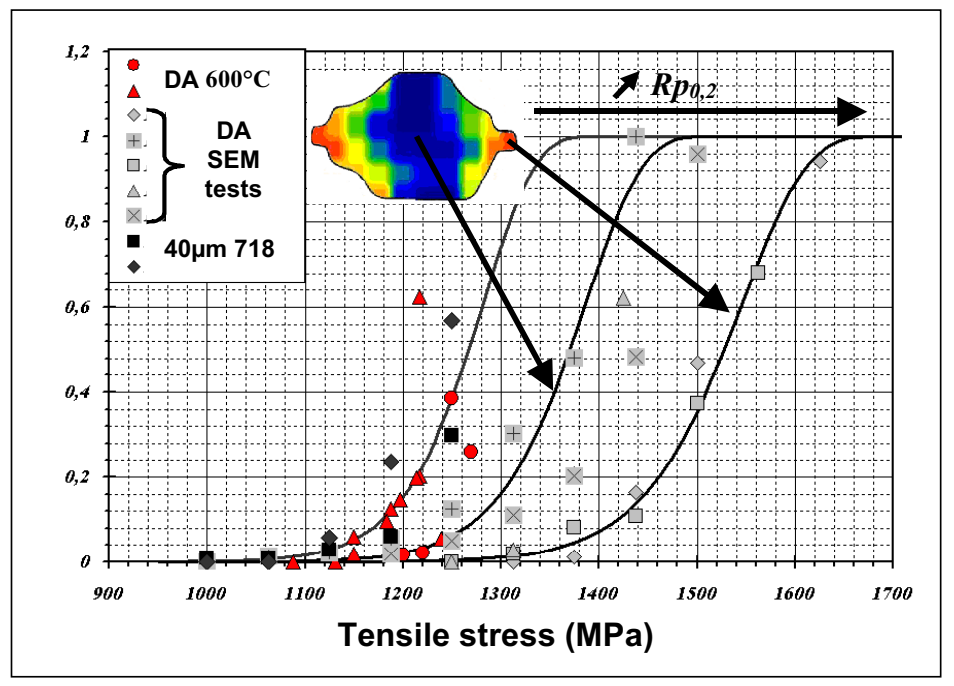

Figure 3. Fraction of cracked $\mathrm{NbC}$ particles vs applied tensile stress, at ambient temperature $(\mathrm{SEM})$ and $600^{\circ} \mathrm{C}$, for two grain sizes and several positions in a turbine disk.

Figure 3.

Figure 3 shows that the proportion of cracked $\mathrm{NbC}$ particles (carbides) increases with the load applied at the first LCF cycle, and also that this fraction depends on the plastic behavior of the alloy characterized: for a given tensile stress applied, the fraction of cracked carbides decreases when the yield strength $\left(R_{0,2}\right)$ increases. This is illustrated in Figure 3.

Figure 3 with two different zones in the initial forged turbine disk: material from the central zone of the disk exhibits a lower yield strength compared to the material taken from the peripheral zones, more hardened by forging thermomechanical process. The same effect is observed with larger grain sizes $(40 \mu \mathrm{m}$ here), obtained from the initial DA718 by an appropriate heat retreat: this material shows lower yield strength, hence higher fractions of cracked carbides.

As a consequence, two initiation micro-mechanisms have been identified in DA718: classical stage I initiation on PSB, observed after a given number of cycles to initiation $\mathrm{N}_{\mathrm{i}}$, and instantaneous but statistical micro-cracking of second phase particles (mainly carbides), increasing with applied tensile stress applied and decreasing with material strength. The two mechanisms are schematically shown in Figure 4 [4]. 


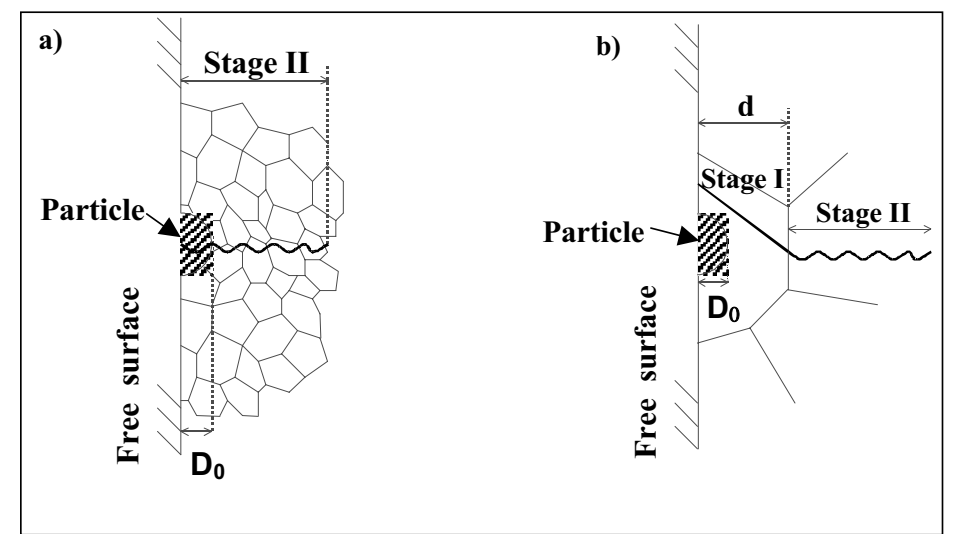

Figure 4. Schematic representation the two fatigue crack initiation types [4]:

a) particle induced initiation (grain size $\mathrm{d}$ smaller than particle size $\mathrm{D}_{0}$ ),

b) Stage I initiation (grain size larger than particle size).

The reason why fatigue crack initiation can occur on sub-surface and surface particles, depending on the applied load, is the higher number of particles available in the sub-surface volume compared to the particles coming out to the surface: when the load is low enough, the number of cracked surface carbides will not be statistically sufficient, and sub-surface initiation sites will become predominant. This effect will be detailed in the next sections.

\section{$\underline{\text { Fatigue Crack Propagation }}$}

Since micro-crack initiation can occur at the first LCF cycle, characterization of micro-crack propagation in DA718 becomes a matter of primary importance. Small fatigue crack propagation measurements have been carried out on DA718 alloy at temperatures ranging from ambient to $600^{\circ} \mathrm{C}$, on a LCF test rig. The propagation of cracks initiated on an artificial defect (created by electro-discharge machining) was monitored using a high focal distance optical microscope (QUESTAR) with a resolution of about $5 \mu \mathrm{m}[2,3]$. Some results are shown in Figure 5, where three testing conditions are reported: the first two correspond to deformation-controlled tests $\left(\mathrm{R}_{\varepsilon}=0\right)$; the third one corresponds to a stress-controlled test $\left(\mathrm{R}_{\sigma}=-1\right)$. Further results are shown elsewhere [3].

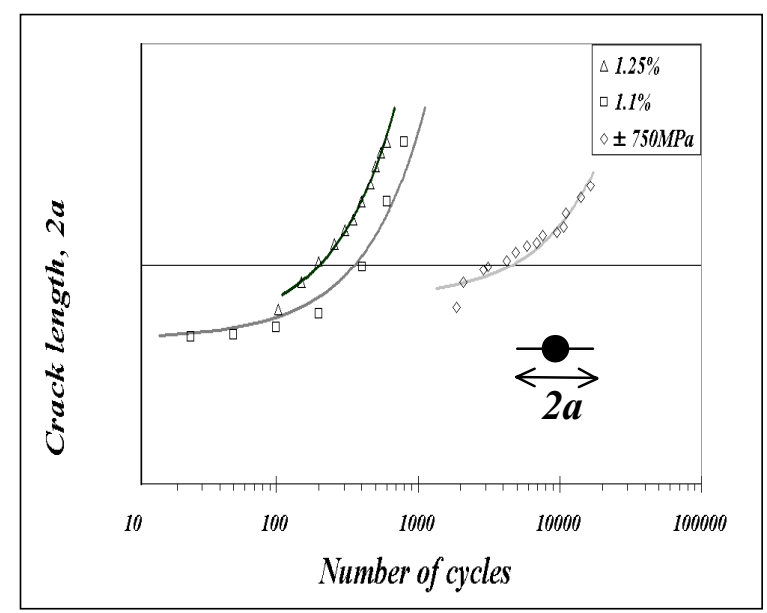

Figure 5. Measured crack length vs number of cycles for three test conditions.

DA $718,600^{\circ} \mathrm{C}[2,3]$. 
Crack length ranges were characterized from a few tens of microns to a few hundreds of microns: in this range of length, it appears that crack length increases following an exponential law, which means that crack propagation rate $d a / d N$ is proportional to crack length $a$. These observations justify the use of the Tomkins crack propagation model proposed in the next section.

\section{Probabilistic life model for DA 718}

Both crack initiation micro-mechanisms and micro-propagation behavior identified for DA 718 and described in the previous section have been modelled at mesoscopic scale $[2,3]$. In order to account for dispersion due to the particle presence in a given volume and the proportion of cracked particles as a function of applied load, a probabilistic formulation has been adopted. The aim of this section is to describe the formulations chosen, and to precise how were identified the main parameters of mesoscopic and macroscopic models.

\section{Mesoscopic Models}

Three mechanisms have been modelized at mesoscopic levels (see [2,3] for further details):

- Stage I micro-initiation (case $b$ in Figure 4): the model chosen here is a Tanaka-Mura type formulation [13] with a slight modification to account for grain size effect, given by equation 1 below, where $N_{i}$ is the number of cycles to initiation, $d$ is the grain size (here, $10 \mu \mathrm{m}), \Delta \varepsilon_{p}$ is the amplitude of plastic strain in the grain (in our case, macroscopic plastic strain calculated by a viscoplastic behavior law is used), and $\mathrm{A}_{\text {Stager }}$ a constant depending on temperature.

$$
N_{i}=\frac{1}{d} \frac{A_{\text {stageI }}}{\left(\Delta \varepsilon_{p}\right)^{2}}
$$

- Probabilistic micro-initiation on particles : experimental data can be well represented by a Weibull type law (see equation 2 ), where failure probability $\left(P_{\text {fracture }}\right)$ depends on tensile load and the material yield strength, as shown by experimental results.

$$
P_{\text {fracture }}=1-\exp \left(-\left[\frac{\Sigma_{1}+\lambda<\left(\sigma_{e q}-R p_{0.2}\right)>}{\sigma_{u}}\right]^{m}\right)
$$

The Beremin law [14] proposed here is adapted to a 3D problem by the use of a fracture stress $\sigma_{d}=\Sigma_{1}+\lambda\left(\sigma_{e q}-R_{p 0.2}\right)$, where $\sigma_{d}$ is the critical decohesion stress, $\Sigma_{1}$ is the maximum principal stress, $\lambda$ the particle shape factor and $\sigma_{\text {eq }}$ the equivalent Mises stress. Here, $<\mathrm{X}>=\mathrm{X}$ if $\mathrm{X}>0 \&<\mathrm{X}>=0$ if $\mathrm{X}<0$.

- Micro-crack propagation: experimental data shown in Figure 5 indicate that crack propagation rate can be considered proportional to crack length, for this range of crack lengths and test conditions. The use of Tomkins model [15], where $d a / d N=\alpha . a$, appears therefore to be justified. Expression of $\alpha$ coefficient is given in Equation 3.

$$
\alpha=\frac{\pi^{2}}{8} \frac{\Delta \varepsilon_{p} \Delta \sigma^{2}}{(2 T)^{2}}\left(1+\frac{\pi^{2}}{8}\left(\frac{\Delta \sigma}{2 T}\right)^{2}\right)
$$


$\alpha$ depends on plastic strain amplitude $\Delta \varepsilon_{p}$, maximum principal stress amplitude $\Delta \sigma$, and a parameter called $\mathrm{T}$ associated to ultimate tensile stress in the plastic zone. Apart from mechanical behaviour, $\mathrm{T}$ is the only parameter to be identified for this model.

\section{Macroscopic Model}

The proposed macroscopic model takes into account the former three mesoscopic models to calculate the number of cycles to failure, $N_{\text {failure }}$, associated to the two possible initiation sites : grains and particles. $N$ failure is the sum of the number of cycles to micro-initiation of a crack with length $d$ (stage I initiation) or $\mathrm{D}_{0}$ (particle size in particle initiation), and the number of cycles to propagate this micro-crack until fracture of the structure (by reaching $\mathrm{K}_{\mathrm{Ic}}$ ). Competition is treated by taking the intiation site leading to minimum $N_{\text {failure }}[2,4]$.

Experimental data indicate that crack initiation on particles has to be treated by a probabilistic approach for at least two reasons:

- Only a fraction of present particles is fractured for a given load (this point is treated by the mesoscopic model presented above)

Depending on strained surface and volume, the number of particles will vary and the probability of particle presence as well. This will produce a scale effect.

\section{Probability of Particle Presence}

The latter probabilistic effect has been thoroughly studied to treat initiation on ceramic inclusions in powder metallurgy superalloys (see e.g. [16,17]). A similar approach is developed here, based on particle counts carried out on DA718 coupons taken from disks: several thousands of particles were counted, and the particle distribution obtained is represented in the normalized curve of Figure 6. Each particle is described by a small sphere (or disk in a 2D description) of equivalent area; the diameter of this sphere (or disk) is plotted in Figure 6.

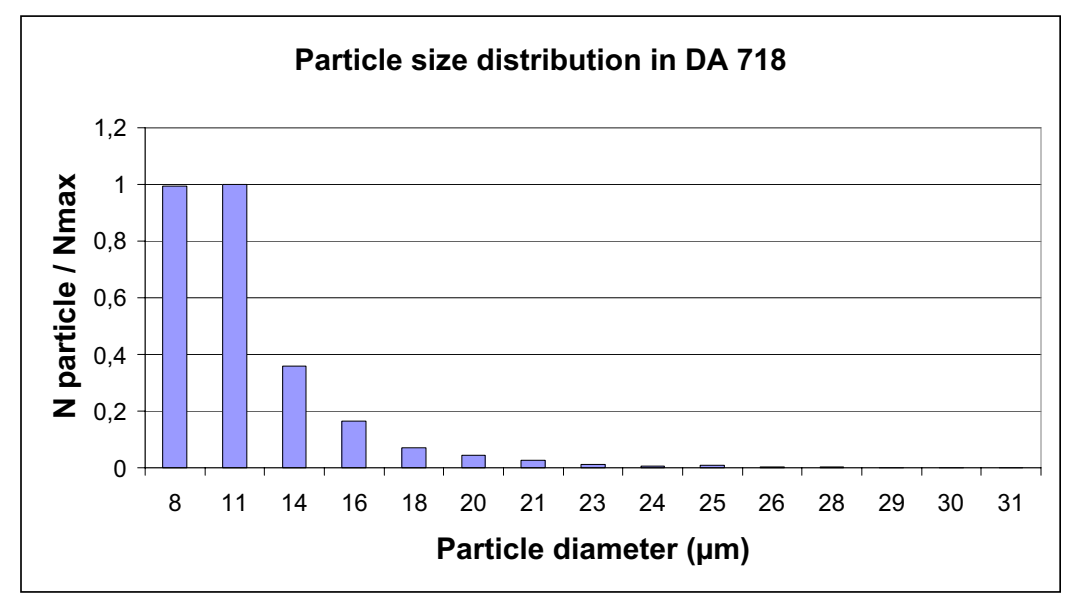

Figure 6. Normalized particle distribution for DA 718.

Knowing this distribution, one can determine the number of particles with diameter $D$ greater than a given diameter $D_{0}, N\left(D_{0} \leq D\right)$, and the associated probability:

$$
P\left(D_{0} \leq D\right)=\frac{N\left(D_{0} \leq D\right)}{N\left(D_{0} \leq D_{\min i}\right)}=\frac{\sum_{D j=D 0}^{D \max i} N\left(D_{0}=D_{j}\right)}{\sum_{D j=D \min i}^{D \max i} N\left(D_{0}=D_{j}\right)}
$$


For identification sake, it is assumed that $\mathrm{P}\left(\mathrm{D}>=\mathrm{D}_{0}\right)$ follows a Poisson law (see [16]). Thus $\mathrm{P}\left(\mathrm{D}>=\mathrm{D}_{0}\right)$ is identified as:

$$
P\left(D \geq D_{0}\right)=1-\exp \left(-N\left(D \geq D_{0}\right)\right)
$$

\section{Global Probability of Failure}

The aim of the model proposed here is to determine a probability of disk failure (presence of a macroscopic crack in the structure) before a given number of cycles. The current certified methods cover such a risk, the probability of failure $P\left(N_{f} \leq N\right)$ being $1 / 1000$, with $N_{f}$ the number of cycles calculated, and $\mathrm{N}$ the given number of cycles for which the risk is calculated.

To determine such probability, statistical dispersion must be taken into account: this is done in the proposed approach thanks to the probability of particle presence, depending on the strained volume or surface, and the probability of particle fracture $P_{\text {fracture }}$.

If one considers only one particle, failure will be achieved if two independent events occur: initiation of a micro-crack at the first cycle (given by $P_{\text {fracture }}$ ), and particle diameter $\mathrm{D}_{0}$ large enough for the crack to reach the final critical size in $\mathrm{N}$ cycles. This second condition is evaluated by integration of Tomkins model between $\mathrm{D}_{0}$ and $\mathrm{D}_{\mathrm{f}}$. This is simplified ultimately according to equation 6 .

$$
P\left(N_{\text {prop_particle }} \geq N\right)_{1 \text { part }}=P\left[\frac{1}{\alpha} \ln \left(\frac{D f}{D_{0}}\right) \geq \frac{1}{\alpha} \ln \left(\frac{D f}{D}\right)\right]=P\left(D_{0} \leq D\right)
$$

The probability to be calculated then corresponds to the presence of a particle of size greater than a given $\mathrm{D}_{0}$. This probability varies with the particle position (surface, sub-surface, internal) and the associated volume.

In the end, the global failure probability is obtained by considering all the particles in the simulation volume, and for all possible particle positions. In this case, according to the weakest link theory, independent probabilities to be multiplied are survival probabilities, $\mathrm{P}_{\text {survival }}=1$ $\mathrm{P}_{\text {failure. }}$

This global failure probability can be calculated (see equation 7) as a post-processing routine of a finite element structural model, with:

- $\quad \mathrm{N}_{\text {part_s }}$ and $\mathrm{N}_{\text {el_surf }}$ the number of particles intercepting the element free surface, and the number of elements at the surface of the structure, respectively;

- $\mathrm{N}_{\text {part_vi }}$ and $\mathrm{N}_{\mathrm{el} \_ \text {internal_vol }}$ the number of particles contained by the volume of the element, and the number of internal elements of the structure, respectively;

- $\quad \mathrm{N}_{\text {part_vss }}$ and $\mathrm{N}_{\text {el_sub_surface }}$ the number of particles contained by the sub-surface volume of the element, and the number of elements at the surface of the structure, respectively.

$$
\begin{aligned}
& P(N f \geq N)_{\text {global }}= \\
& 1-\prod_{s=1}^{\text {Nél_surf }}\left[\left(1-P_{\text {fracture }}\left(\sigma_{d}\right) * P\left(D_{0} \leq D\right)\right)^{\text {Npart_s }}\right] * \prod_{v=1}^{\text {Nél_internal_vol }}\left[\left(1-P_{\text {fracture }}\left(\sigma_{d}\right) * P\left(D_{0} \leq D\right)\right)^{\text {Npart_vi }}\right] \\
& * \prod_{v=1}^{\text {Nél_sub-surface }}\left[\left(1-P_{\text {fracture }}\left(\sigma_{d}\right) * P\left(D_{0} \leq D\right)\right)^{\text {Npart_vss }}\right]
\end{aligned}
$$




\section{Parameter Identification}

Due to the reduced number of parameters, a correct description of DA718 viscoplastic behavior is necessary to use this model. Chaboche-type behavior laws, including viscosity and both nonlinear isotropic and non-linear kinematic hardening, were identified for this purpose at $450^{\circ} \mathrm{C}$ and $600^{\circ} \mathrm{C}$ [18]. Use of thresholds in the non-linear kinematic hardening laws allowed to describe correctly mean stress effect. Nevertheless, differences still exist between measured and calculated plastic deformation. This gap obliged to identify model parameters based on calculated behavior rather than measured behavior, to avoid a bias in validation presented in the next section.

The parameters introduced in mesoscopic and macroscopic models were identified by specific testing when possible (e.g., micro-crack propagation presented in previous section to identify Tomkins law's T parameter), or by global identification on the whole LCF DA718 database available at Snecma. $T$ parameter requires particular attention, since Tomkins law does not account for positive mean stress effects. This difficulty was solved by making $\mathrm{T}$ parameter dependent on mean stress, and verifying this assumption by tests conducted under appropriate conditions.

\section{Model validation : notched LCF specimens}

In order to validate the global macroscopic method, one last aspect has to be considered: the existence, in disk structures, of notched areas and subsequent stress gradients. This method could then be validated on a complete structure. However, no Snecma turbine disk has ever shown hopefully- any crack, so experimental validation data would be very limited. As a consequence, LCF tests were carried out on "mini-structures", notched samples, with two types of notch, described here as "sharp" (more representative of disk dovetails, with a root radius of $1 \mathrm{~mm}$ and a stress concentration factor Kt of 2.5) and "smooth" notch (more representative of disk bores, with a root radius of $4.5 \mathrm{~mm}$ and a stress concentration factor $\mathrm{Kt}$ of 1.3). These notched samples were then calculated by FEA, and the proposed method was applied as a post-processing routine, and finally compared to the results given by the current certified (or "standard") lifing method.

\section{Experimental data}

LCF tests on notched specimens were performed at two temperatures corresponding to those observed in turbine disks, and several stress levels were applied, with a focus on one level to multiply data under the same testing condition and to acquire more visibility on scatter. Tests were load-controlled, carried out at a frequency of $1 \mathrm{~Hz}$. All samples were taken from the same turbine disk to limit scatter due to material origin, but various zones of the disk with different mechanical behaviors were tested.

Figure 7 summarizes the results of these LCF tests. The existence of two fatigue curves is due to the differing notch factor between the two sample geometries, and the fact that second coordinate of the graph is stress amplitude applied to the test sample, away from the notch. 


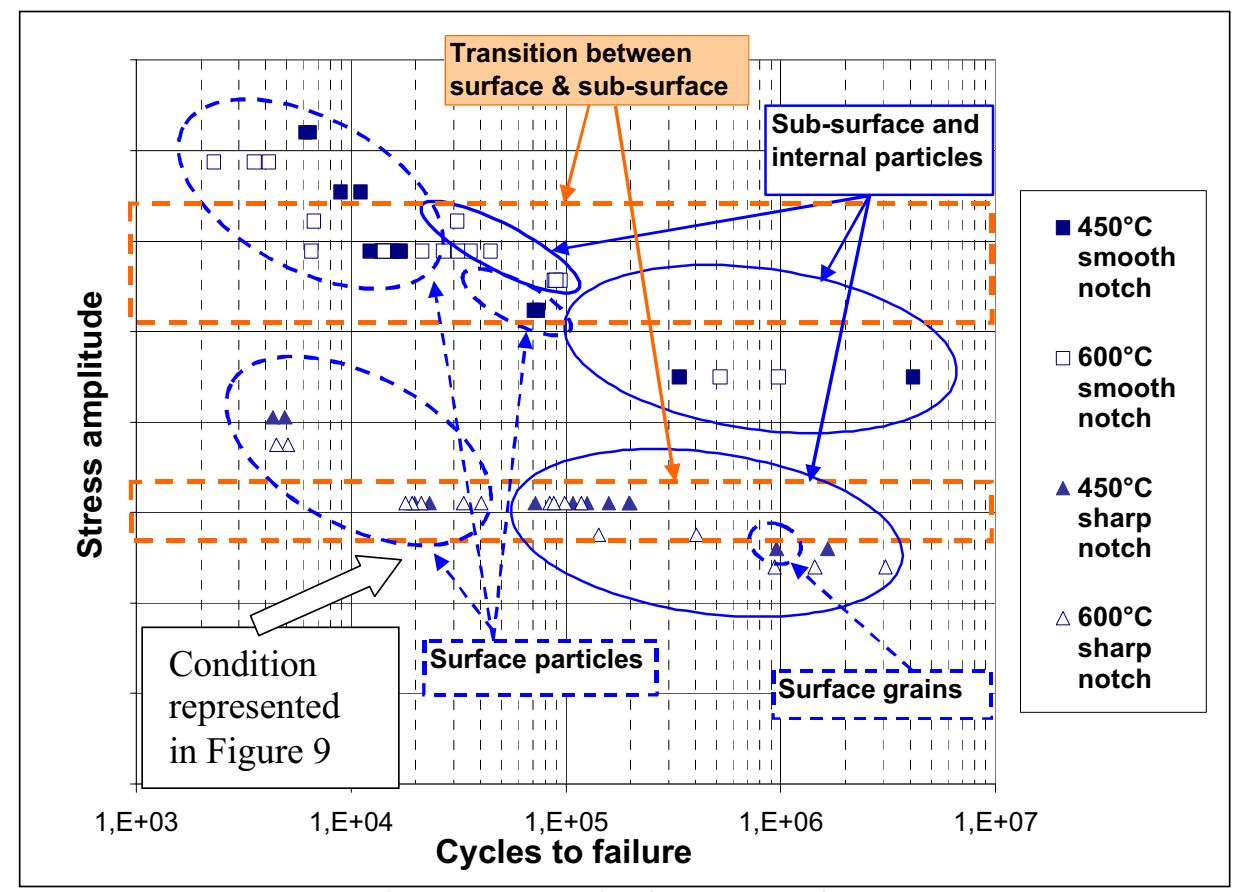

Figure 7. Notched LCF results

In this figure, one can observe on this figure that for both geometries, LCF results are very close for $450^{\circ} \mathrm{C}$ and $600^{\circ} \mathrm{C}$ tests, which can be explained by the fact that DA 718 mechanical properties show very little variation in this range of temperatures.

In both cases, three domains appear:

- At high loads, a domain with predominant initiation on surface particles;

- At low loads, a domain with predominant initiation on sub-surface or internal particles, and seldom stage I initiation (surface grains)

- At intermediate loads, a transition domain where all initiation sites coexist, leading to a significant scatter in the results.

This last remark justifies the choice of intermediate load to multiply test samples, in order to validate the global method on a case with significant LCF dispersion and various possible initiation sites.

\section{Finite Element Analysis}

Finite Element Analysis (FEA) was carried out on plane strain 2D models of the notched samples, with Abaqus ${ }^{\circledR}$ coupled with Zebulon ${ }^{\circledR}$, which allowed use of the mechanical behavior laws described in the previous section. Mesh was refined in the notch area, and a parametric study was carried out to determine the mesh size leading to stress convergence. The mesh size obtained is $0.1 \mathrm{~mm}$. 300 cycles were modelized, leading to stabilization of stresses and strains.

Post-processing routines corresponding to mesoscopic and macroscopic models mentioned before were implemented at this stage:

- $P_{\text {fracture }}$ was post-processed from stresses calculated at the first LCF cycle;

- $T$ parameter and Tomkins law's $\alpha$ parameter were post-processed from stresses and strains calculated at the stabilized LCF cycle.

Figure 8 represents such results for a sharp notch model submitted to an intermediate stress level. Model geometry has been voluntarily altered. 

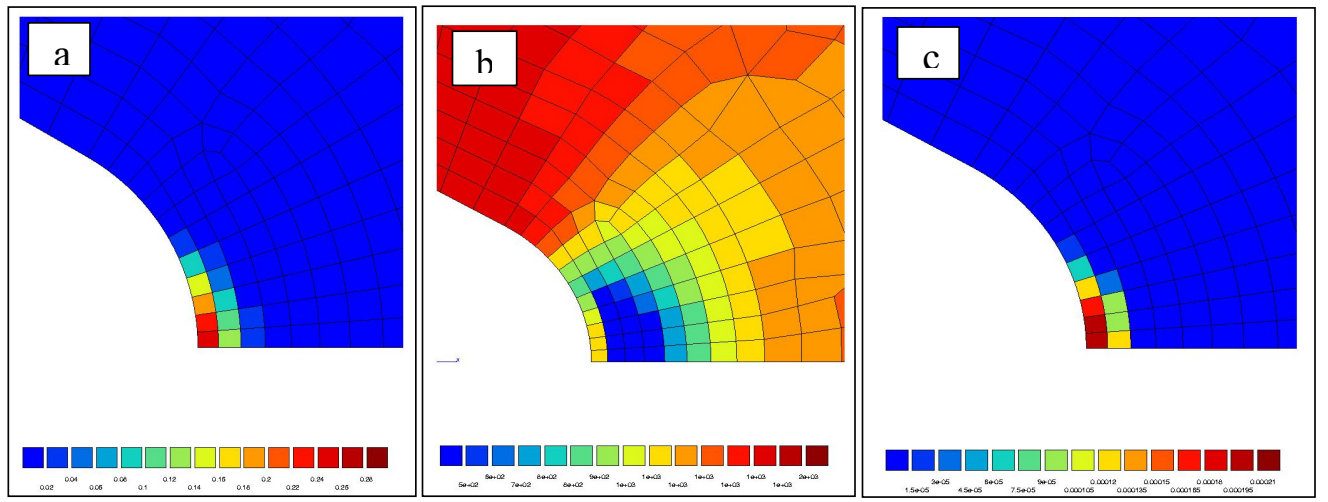

Figure 8. Post-processing realized on a « sharp notch » sample, at intermediate stress level : a) $P_{\text {fracture }}$ calculated at the first LCF cycle, $\left.b\right) T$ parameter and c) $\alpha$ parameter, both calculated at stabilized LCF cycle

These post-processing results show that the most critical area concerning $P_{\text {fracture }}$ and $\alpha$ parameter is located at the surface elements of the notch, even if $T$ parameter is minimum at the elements just below the surface. It should be noted that $\alpha$ parameter depends indeed essentially on $\Delta \varepsilon_{p}$.

\section{Comparison Between Model and Experimental Data}

Post-processed data, averaged for each element of the modeled structure, are treated by the global probabilistic method, giving global $P_{\text {failure }}$ as a function of a given number of applied cycles, N. Results of the proposed method are given in Figure 9 for the same geometry and same level as in Figure 8. Experimental failure probabilities are obtained here by ranking all experimental data by ascending numbers of cycles to failure, then attributing a "probability" $\mathrm{P}_{\mathrm{i}}$ given by equation 8 , where $R_{i}$ is the rank for the $i^{\text {th }}$ test data.

$$
P_{i}\left(N_{f} \leq N\right)=\frac{R_{i}}{1+\max _{i}\left(R_{i}\right)}
$$

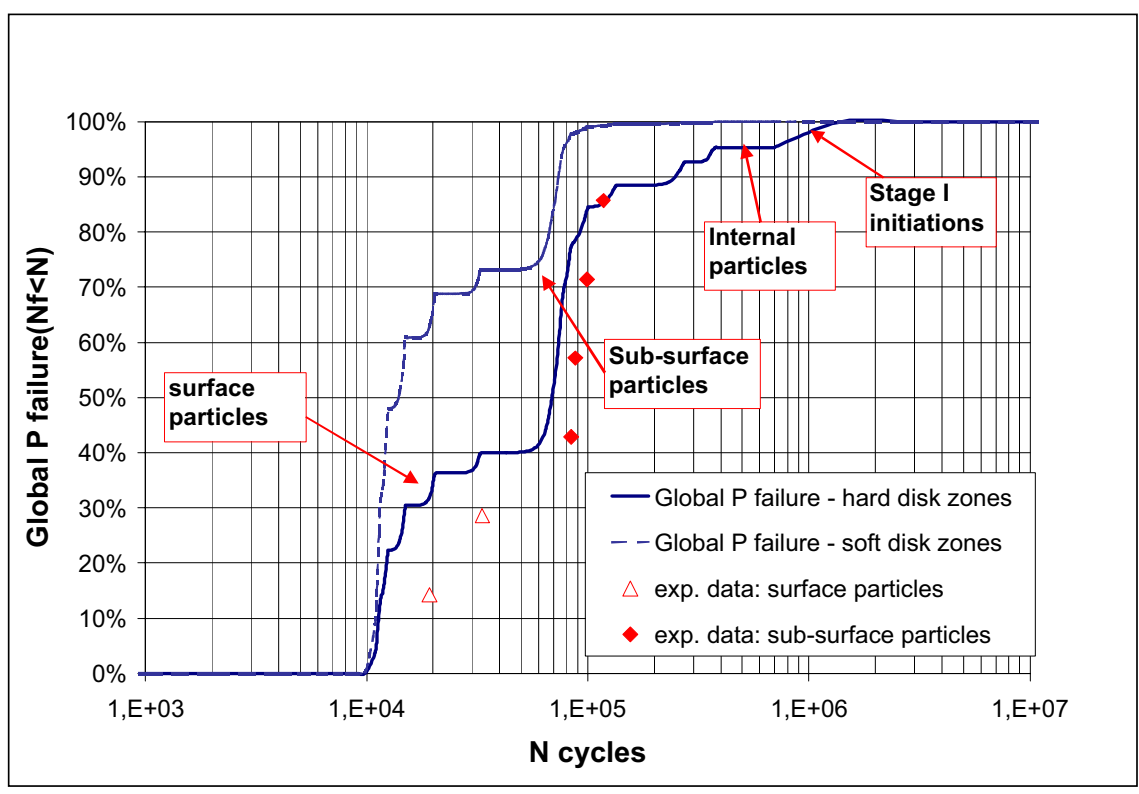

Figure 9. Global Probability of failure calculated for a sharply notched sample tested at intermediate stress, $600^{\circ} \mathrm{C}$. 
In this graph, two calculated curves appear; this is due to the differences of mechanical behavior identified for various original locations of material in the turbine disk: the two curves represent the lower and upper limits due to the variability in strength level of the material. Such a graph has to be analyzed as regards not only predicted lives, but also Ncycle range covered between Pfailure $=0 \%$ and $\mathrm{P}$ failure $=100 \%$, which corresponds here very well to experimental data scatter. Predominant initiation sites are also reported, and one can see that predicted sites correspond to experimental data, with a transition predicted from the model, from surface particles (for shorter lives) to sub-surface and internal particles (for longer lives). Curve irregularities indicate "jumps" when one element starts to be critical in the analysis: these irregularities would be softened using a more refined mesh.

Thus the global probabilistic model shows a very good agreement with experimental data (with predicted lives slightly shorter than experimental ones) for this intermediate stress level. To end with validation of the model, Figure 10 shows a comparison of the results obtained from the proposed probabilistic model and results of the "standard" certified method, along with the integrality experimental data gathered on sharp notch geometry at $600^{\circ} \mathrm{C}$. Both mean life (i.e. life calculated for $\mathrm{P}_{\text {failure }}=50 \%$ ) and minimum life (i.e. life calculated for $\mathrm{P}_{\text {failure }}=0.14 \%$ ) are represented for both methods.

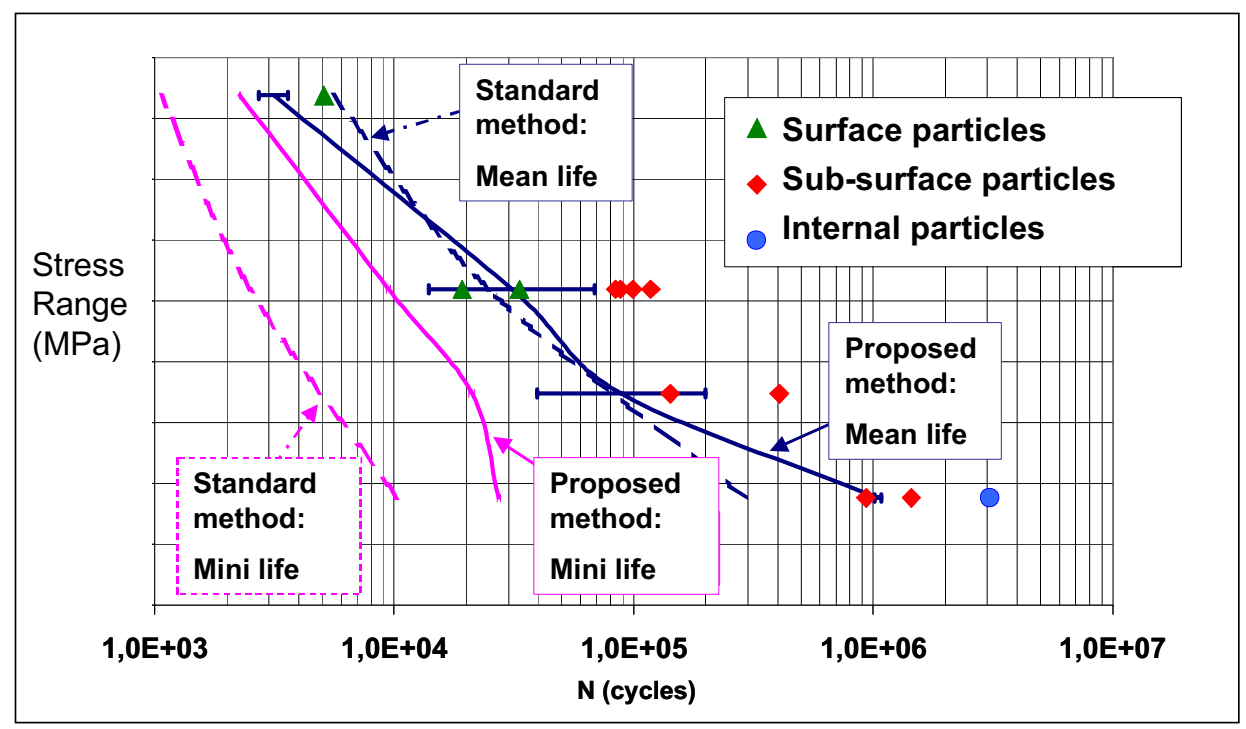

Figure 10. Comparison between experimental data, standard method and proposed method. Simulation of a sharply notched sample loaded at all experimental levels available.

This figure shows again a very good agreement of mean and minimum lives calculated by the probabilistic method as compared to experimental data. At low stress level, mean life is better described by probabilistic method than standard method. Moreover, minimum lives calculated with the proposed probabilistic method are greater than those given by standard method, and keep shorter than the shortest experimental life at the corresponding level. However, to validate this result much more experimental data would be necessary.

\section{Conclusion}

The micro-mechanisms of fatigue crack initiation and propagation identified for DA 718 alloy, as well as the competition between initiation site types and locations, have been modelized by mesoscopic models using very few parameters and depending strongly on material mechanical 
behavior. LCF data scatter was mainly attributed to probabilistic nature of particle presence in a given volume, and probability of fracture at the first LCF cycle.

In order to describe these effects and to propose a model able to account for LCF scatter by the only means of physical mechanisms description, a global macroscopic and probabilistic model has been proposed. This model can be used as a post-processing of a finite element analysis.

Confrontation of the global probabilistic model to experimental data obtained on notched samples give good results, and the proposed method shows more realistic results than the standard one when applied to notched specimens. Contrary to the standard method, it allows in addition to determine the predominant initiation sites as a function of loading, material behavior and microctructure inherited form forging process.

Nevertheless, this first proposed method can be much improved, by a formulation less dependent on calculated plastic behavior, better adapted to gradient stresses, and taking into account other sources of experimental scatter, such as stage I initiation (for IN718 with larger grain sizes).

\section{Acknowledgements}

This work was supported by the French Ministry of Research through FAMICRO programme.

\section{References}

1. D. Fournier, A. Pineau, "Low cycle fatigue behavior of Inconel 718 at $298 \mathrm{~K}$ and 823K” Metallurgical Transaction A. 8 (1977), 1095-1105.

2. F.Alexandre, Probabilistic and microstructural aspects of fatigue crack initiation in IN718 (Ecole des Mines de Paris PhD Thesis, 2004).

3. F.Alexandre, S.Deyber, A.Pineau, "Modelling the optimum grain size on the low cycle fatigue life of a Ni based superalloy in the presence of two possible crack initiation sites", Scripta Materialia, 50 (2004), 25-30.

4. Y. C. Fayman, "Microstructural Characterization and Elemental Partitioning in a Directaged Superalloy (DA718)”, Materials Science and Engineering, 92 (1987), 159-171.

5. S.P. Lynch et al., "Fatigue crack growth in Nickel-base superalloys at 500-700 ${ }^{\circ} \mathrm{C}$. II: DirectAged alloy 718”, Fatigue Fract. Eng Mater. Struct., 17-3 (1994), 313-325.

6. J.Y. Guédou, G. Simon, J. M. Rongvaux, "Development of damage tolerant Inco 718 for high temperature usage" Proceedings Superalloys 718, 625, 708 and various derivatives (1994), 509-522.

7. L. A. James, "The effect of grain size upon the fatigue-crack propagation behavior of alloy 718 under hold-time cycling at elevated temperature", Engineering Fracture Mechanics, 253 (1996), 305-314.

8. J. P. Pedron, A. Pineau, "The effect of Microstructure and Environment on the Crack Growth Behaviour of Inconel 718 Alloy at $650^{\circ} \mathrm{C}$ under Fatigue, Creep and Combined Loading", Materials Science and Engineering, 56 (1982), 143-156. 
9. T. Connolley, P. A. S. Reed, J. M. Starink, "Short crack initiation and growth at $600^{\circ} \mathrm{C}$ in notched specimens of Inconel 718", Materials Science and Engineering, A 340 1-2 (2003), 139-154.

10. N. Späth et al., "718 superalloy forging simulation: a way to improve process and material potentialities", Proceedings Superalloys 718, 625, 706 and Various Derivatives (2001), 173183.

11. S. Suresh, Fatigue of Materials Second Edition (Cambridge University Press, 1998).

12. A. Pineau, "Short fatigue crack behavior in relation to three-dimensional aspects and crack closure effect”, Small fatigue cracks, ed. R.O. Ritchie, J. Lankford, AIME (1986), 191-210.

13. K. Tanaka and T. Mura, "A dislocation model for fatigue crack initiation", Journal of Applied Mechanics, Vol. 48 (1981), 97-103.

14. F.M. Beremin, "Cavity formation from inclusions in ductile fracture of A508 steel", Metallurgical Transactions A, Vol. 12, N¹7 (1981), 723-731.

15. B. Tomkins, "Fatigue crack propagation - An Analysis", Philosophical Magazine, Vol. 18 (1968), 1041-1066.

16. A. de Bussac and J. C. Lautridou, "A probabilistic model for prediction of LCF surface crack initiation in PM alloys", Fatigue and fracture of engineering materials and structures, Vol. 16, n॰8 (1993), 861-874.

17. A.Pineau, "Superalloy discs durability and damage tolerance in relation to inclusions", High Temperature Materials for Power Engineering, Part II, ed. E.Bachelet et al. (1990), 913922.

18. J. Lemaitre, J.L. Chaboche, Mécanique des Matériaux, ed. Dunod (1989). 